\title{
Clues to the Pathogenesis of Melasma from its Histologic Findings
}

Soon-Hyo Kwon ${ }^{1,2}$ and Kyoung-Chan Park ${ }^{1,2 *}$

${ }^{1}$ Seoul National University, College of Medicine, Korea

${ }^{2}$ Seoul National University, Bundang Hospital, Gyeonggi, Korea

\begin{abstract}
Melasma is a common acquired hypermelanosis that affects sun-exposed areas of the skin, especially the face. Its histologic manifestations are evident in the epidermis, extracellular matrix, and dermis. One of the hallmarks of melasma is an increase in the amount of epidermal melanin; however, whether melanocyte numbers increase or not is a topic of debate. Interestingly, basement membrane abnormalities also characterize melasma. Furthermore, solar elastosis is recognized as one of the dermal pathologic findings of melasma. These findings suggest that extracellular matrix abnormalities are consistently found in melasma. In the dermis, increased vascularity and increases in mast cell numbers are observed, indicating that dermal factors have important roles in the pathogenesis of melasma, despite melasma being characterized by epidermal hyperpigmentation. This review discusses these histologic characteristics of melasma, and it considers their implications for the pathogenesis of this skin condition.
\end{abstract}

Keywords: Basement membrane; Histopathology; Mast cells, Melasma; Vascularization

\section{Introduction}

Melasma is an acquired hypermelanosis characterized by the development of symmetrical, irregular light-to-dark brown macules and patches on sun-exposed areas of the skin, especially on the skin of the face [1]. It is common among Asian and Hispanic women who are in their third or fourth decades of life [2]. Three patterns of melasma are recognized clinically that are based on the distribution of the hyperpigmentation on the face, namely, the centrofacial, malar, and mandibular patterns. However, melasma often presents as a mixture of these patterns. Factors involved in the pathogenesis of melasma include a genetic predisposition, chronic exposure to ultraviolet (UV) radiation, and female sex hormones [3-6]. However, the pathogenesis of melasma has not yet been fully elucidated.

The histopathologic features of melasma skin might provide clues towards understanding its pathogenesis. This review discusses five histologic characteristics of melasma, namely, epidermal hyperpigmentation, basement membrane disruption, solar elastosis, increased vascularization, and a high prevalence of mast cells, and it considers their implications for the pathogenesis of melasma.

\section{Epidermal hyperpigmentation}

The most characteristic histologic feature of melasma is the increase in the amount of melanin in the epidermis. Fontana-Masson staining has shown that, the melanin content of melasma skin is higher in all layers of the epidermis, including the stratum corneum, than that in perilesional normal skin [7-9]. Image analysis of Fontana-Massonstained sections of skin from 22 patients with melasma showed a significant difference in the density of melanin between melasma skin (mean \pm standard deviation $[S D]: 0.37 \pm 0.02$ ) and perilesional normal skin $(0.34 \pm 0.02)(p<0.01)$ [10]. These findings indicate that the development of melasma involves accelerated melanin synthesis, increased levels of melanin transfer to the keratinocytes, and reduced melanin degradation.

Reports on melanocyte numbers in melasma are inconsistent. Kang et al. [7] found a higher melanin content and increased numbers of melanocytes in melasma skin. Their study involved the quantitative image analysis of 56 Fontana-Masson-stained sections. They showed that, compared with perilesional normal skin, the number of melanocytes per millimeter of epidermal length and the number of melanocytes per millimeter of rete ridge length increased by $24 \%$ and $27 \%$, respectively, in melasma skin, while the pigmented area per millimeter of epidermal length and the pigmented area per millimeter of rete ridge length increased by $73 \%$ and $39 \%$, respectively. In addition, ultrastructural observations that included clear increases in the numbers of melanosomes and melanocytes in melasma skin were reported. In contrast, a study by Grimes et al. [11] of 22 skin specimens from subjects with Fitzpatrick skin types IV-VI immunostained using Mel-5, did not find a significant increase in melanocyte numbers in melasma skin compared with perilesional normal skin. Moreover, in their study of 44 patients with melasma, Miot et al. [10] did not find any differences in melanocyte numbers between melasma skin and perilesional normal skin sections labeled using a Melan-A antibody.

Electron microscopy has shown higher numbers of mature melanosomes in keratinocytes and melanocytes in melasma skin [10], and a significantly higher number of dendrites per keratinocyte in melasma skin $(7.55 \pm 2.53$ dendrites per keratinocyte) than that in perilesional normal skin $(5.28 \pm 1.85$ dendrites per keratinocyte $)$ $(p<0.05)$ [11]. Furthermore, electron microscopy demonstrated increased levels of activity within melanocytes in melasma skin, which was deduced from the presence of higher organelle numbers, including mitochondria, Golgi apparatuses, rough endoplasmic reticula, and ribosomes [7].

Immunohistochemistry using NKI-beteb, which recognizes the melanocyte lineage-specific pmel-17 antigen, showed a higher staining intensity in melasma skin than in normal skin (Figure 1). Compared with nonlesional skin, increased expression of tyrosinase has been demonstrated [12]. Mel-5 immunostaining, which detects tyrosinaserelated protein (TRP)-1, also increased in intensity in melasma skin than in normal skin, suggesting that levels of TRP-1 are higher in melasma melanocytes [7]. In addition, we have observed the elevated expression of TRP-2 in melasma skin (Figure 1). These findings support the concept of an increased level of melanogenesis in the pathogenesis of melasma.

*Corresponding author: Kyoung Chan Park, MD, Department of Dermatology, Seoul National University College of Medicine, Seoul National University, Bundang Hospital, 300 Gumi-dong, Bundang-gu, Seongnam-si, Gyeonggi-do 463-707, Korea Tel: +82-31-787-7311; Fax: +82-31-787-4058; E-mail: gcpark@snu.ac.k

Received: July 08, 2014; Accepted: October 17, 2014; Published: October 19, 2014

Citation: Kwon SH, Park KC (2014) Clues to the Pathogenesis of Melasma from its Histologic Findings. Pigmentary Disorders 1:141. doi: 10.4172/JPD.1000141

Copyright: $\odot 2014 \mathrm{Kwon}$ SH, et al. The terms of the Creative Commons Attribution License, which permits unrestricted use, distribution, and reproduction in any medium, provided the original author and source are credited. 


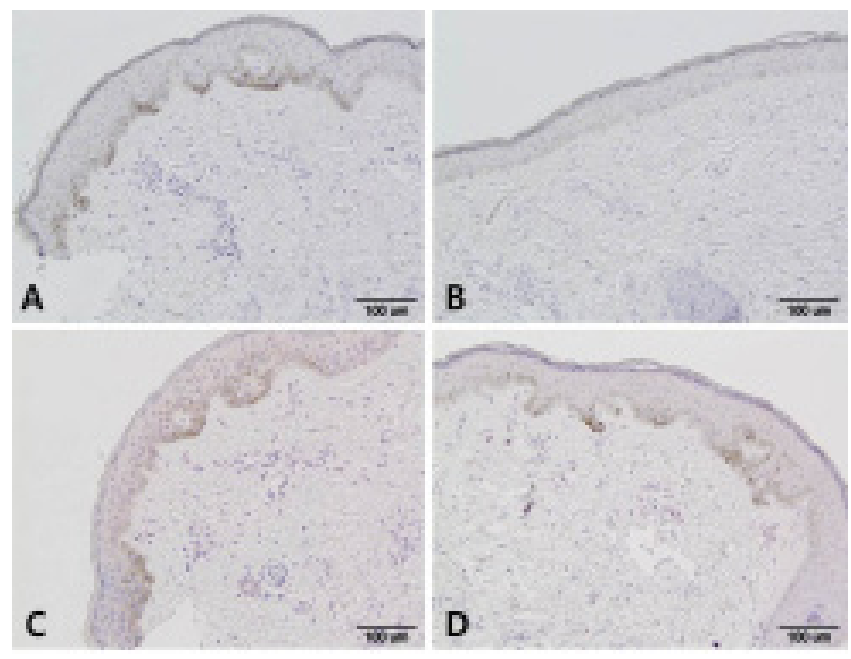

Figure 1: Immunostaining for NKI-beteb and tyrosinase-related protein (TRP)-2 in melasma skin before and after treatment with a combination of soybean extract and niacinamide for eight weeks. Immunostaining for (A) NKI-beteb in melasma skin before treatment $(\times 100)$. (B) NKI-beteb in melasma skin after treatment $(\times 100)$. (C) TRP-2 in melasma skin before treatment $(\times 100)$, and (D) TRP-2 in melasma skin after treatment $(\times 100)$.

\section{Basement membrane disruption}

Some studies have investigated the status of the basement membrane in melasma skin. For example, Sanchez et al. [13] demonstrated the presence of the vacuolar degeneration of the basal cells and the focal vacuolar degeneration of the basement membrane in $3.9 \%(3 / 76)$ of melasma skin specimens. In contrast, Kang et al. [7] did not observe any disruptions of the basement membrane in their evaluation of skin samples from 56 Korean patients with melasma using diastase-resistant periodic acid-Schiff (D-PAS) staining and electron microscopy. However, the same author recently reported that pendulous melanocytes associated with basement membrane abnormalities are a characteristic feature of melasma [14]. Another study of melasma patients with Fitzpatrick skin types IV and V revealed that D-PAS staining and anti-collagen type IV immunohistochemistry indicated damage to the basement membrane in $95.5 \%$ and $83 \%$ of the skin samples, respectively [9]. Basement membrane disruption could be caused by elevated levels of matrix metalloproteinase (MMP)- 2 and MMP-9, which degrade type IV collagen and type VI collagen in the skin during chronic UV exposure [15].

Since free melanin and melanophages are present in the dermis of melasma skin, disruption of the basement membrane could facilitate the descent or the migration of melanocytes and melanin into the dermis $[7,9]$. Histologic findings such as the protrusion of pigmented basal cells into the dermis of $66 \%$ of melasma skin samples compared with that of pigmented basal cells into the dermis of $20 \%$ of photoprotected nonlesional skin samples, support this hypothesis [9]. Consequently, the treatment of melasma is challenging because it is often recalcitrant to therapy and, even after being successfully cleared, it frequently recurs [13].

\section{Solar elastosis}

Solar elastosis is one of the most commonly observed histologic characteristics of melasma skin. Kang et al. [7] reported a moderate-tosevere degree of solar elastosis in $93 \%$ of the melasma patients included in their study. Melasma skin showed a significantly higher degree of solar elastosis than perilesional normal skin ( $83 \%$ vs. $29 \%, p<0.05)[9]$.
Furthermore, the amount of elastotic material was significantly higher in melasma skin than that in perilesional normal skin $(13.3 \pm 2.8 \%$ vs. $10.2 \pm 2.9 \%, p<0.001$ ) [8]. Moreover, thick, highly curled, and more fragmented elastic fibers were observed in Verhoeff-van Gieson-stained sections of melasma skin [7].

The higher level of solar elastosis in melasma skin implies that chronic sun exposure is a prerequisite for the development of melasma. After UVB irradiation, keratinocytes induce melanocyte proliferation and melanogenesis by secreting Stem Cell Factor (SCF), basic Fibroblast Growth Factor (bFGF), interleukin-1, endothelin-1, inducible nitric oxide synthase, $\alpha$-melanocyte-stimulating hormone, and adrenocorticotropic hormone [16-19]. The secretion of prostaglandin E2 after UVB exposure results in larger and more dendritic melanocytes [20]. Furthermore, solar damage of the dermis could induce the secretion of melanogenic cytokines, including SCF and hepatocyte growth factor, from the dermal fibroblasts, thereby influencing the development of hyperpigmentation in the overlying epidermis [21-22].

\section{Increased vascularization}

Accumulating evidence has shown that the number of blood vessels is higher in melasma lesions than in perilesional normal skin [23-25]. An immunohistochemical study of factor VIIIa-related antigen showed a considerable increase in the number of enlarged blood vessels, vessel size, and vessel density in melasma skin compared with perilesional normal skin [23]. The elevated expression of vascular endothelial growth factor (VEGF) in keratinocytes has led to the hypothesis that VEGF may play a role in the behavior of the melanocytes in the skin, because functioning VEGF receptors were demonstrated in melanocytes in vitro [26]. Elevations in the levels of c-kit, SCF, and inducible nitric oxide synthase have also been observed, which could affect vascularization $[27,28]$.

Tranexamic acid (TXA) inhibits plasmin, a key molecule involved in angiogenesis that converts extracellular matrix-bound VEGF into its free forms [29]. TXA has also been reported to suppress neovascularization-induced bFGF [30]. In a recent clinical trial that evaluated the efficacy of systemic TXA in the treatment of melasma, we demonstrated significant decreases in the lesional melanin index and in the erythema index after the oral administration of $250 \mathrm{mg}$ TXA three times per day for eight weeks [31]. Histologic analysis showed significant reductions in the level of epidermal pigmentation and vessel numbers (Figure 2A-D). These findings suggest that the interactions between increased levels of vascularization and the melanocytes may act on the development of hyperpigmentation within the overlying epidermis.

\section{Mast cell prevalence}

Mast cells are observed more frequently in melasma skin than in non-lesional skin, especially in the dermal elastotic areas [31] (Figure $2 \mathrm{E}-\mathrm{F})$. The median prevalence of dermal mast cells was significantly higher in melasma skin than in perilesional normal skin $(173 \pm 57 \%$ vs. $145 \pm 57 \%, p=0.04$ ) [8]. Using an antitryptase antibody, the number of mast cells detected was $58 \pm 39.9$ cells $/ \mathrm{mm}^{2}$ in melasma skin compared with $37 \pm 28.8$ cells $/ \mathrm{mm}^{2}$ in perilesional normal skin $(p<0.04)$ [9].

The role of mast cells in the development of melasma has not been definitively elucidated. Since repetitive UV irradiation induces the production of mast cell tryptase, which degrades type IV collagen, elevated mast cell numbers and tryptase levels could weaken the basement membrane in melasma skin [32]. Mast cells could trigger solar elastosis by inducing the production of elastin by fibroblasts, either directly or via other cell types or cytokines $[33,34]$. Solar elastosis 

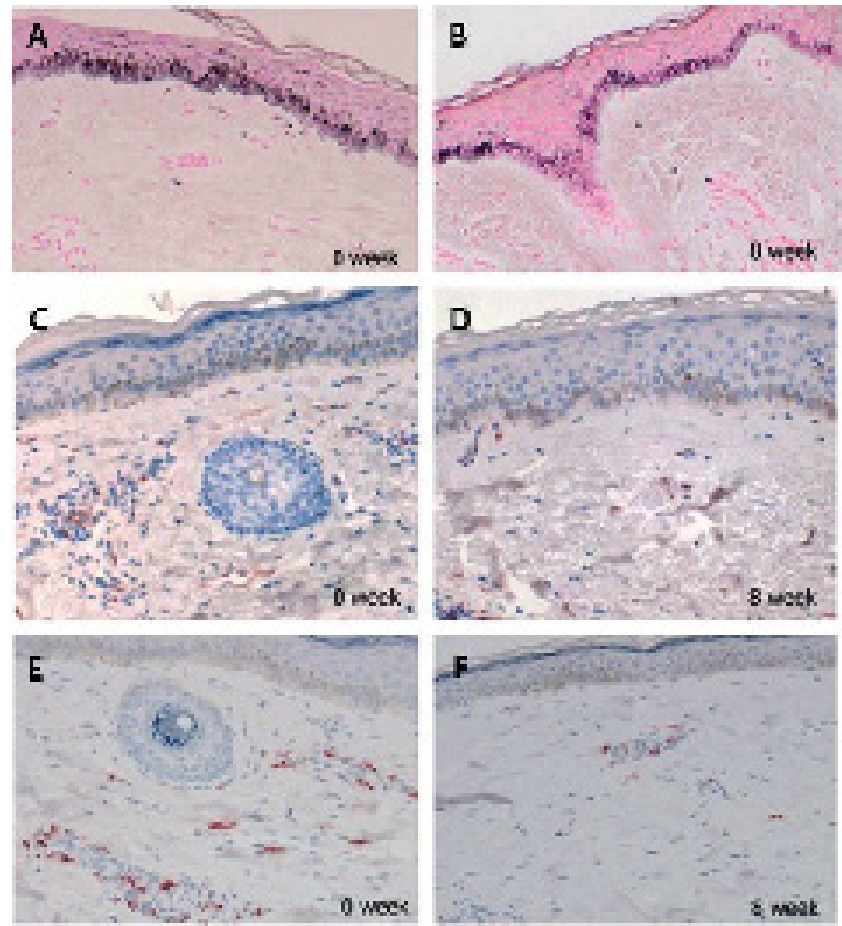

Figure 2: Histologic changes after eight weeks of treatment with tranexamic acid. (A, B) Fontana-Masson staining shows reduced epidermal pigmentation $(\times 100)$. (C, D) Anti-CD31 staining shows reduced levels of vascularity $(\times 100)$. (E, F) Antitryptase staining shows reduced mast cell numbers $(\times 100)$. (Reproduced from $\mathrm{Na}$ et al. [33]).

did not develop in mast cell-deficient mice that were repeatedly irradiated with UV [35]. Elevated numbers of mast cells together with the presence of infiltrating leukocytes and dilated blood vessels might reflect the chronic skin inflammation that underlies the development of melasma [8]. Finally, mast cells can also induce vascular proliferation by secreting angiogenic factors, including VEGF, fibroblast growth factor-2, and transforming growth factor- $\$$ [36].

\section{References}

1. Kwon SH, Park KC (2014) Melasma and common pigmentary dermatoses in Asian individuals and an overview of their treatment. J Clin Investigat Dermatol 2: 8 .

2. Newcomer VD, Lindberg MC, Sternberg TH (1961) A melanosis of the face ("chloasma"). Arch Dermatol 83: 284-299.

3. Pathak MA, Riley FC, Fitzpatrick TB (1962) Melanogenesis in human skin following exposure to long-wave ultraviolet and visible light. J Invest Dermatol 39: 435-443.

4. Grimes PE (1995) Melasma. Etiologic and therapeutic considerations. Arch Dermatol 131: 1453-1457.

5. Ortonne JP, Arellano I, Berneburg M, Cestari T, Chan H, et al. (2009) A global survey of the role of ultraviolet radiation and hormonal influences in the development of melasma. J Eur Acad Dermatol Venereol 23: 1254-1262.

6. Resnik S (1967) Melasma induced by oral contraceptive drugs. JAMA 199: 601-605.

7. Kang WH, Yoon KH, Lee ES, Kim J, Lee KB, et al. (2002) Melasma: histopathological characteristics in 56 Korean patients. Br J Dermatol 146: 228 237.

8. Hernández-Barrera R, Torres-Alvarez B, Castanedo-Cazares JP, Oros-Ovalle $C$, Moncada B (2008) Solar elastosis and presence of mast cells as key features in the pathogenesis of melasma. Clin Exp Dermatol 33: 305-308.
9. Torres-Álvarez B, Mesa-Garza IG, Castanedo-Cázares JP, Fuentes-Ahumada C, Oros-Ovalle C, et al. (2011) Histochemical and immunohistochemical study in melasma: evidence of damage in the basal membrane. Am J Dermatopathol 33: 291-295.

10. Miot LD, Miot HA, Polettini J, Silva MG, Marques ME (2010) Morphologic changes and the expression of alpha-melanocyte stimulating hormone and melanocortin-1 receptor in melasma lesions: a comparative study. Am J Dermatopathol 32: 676-682.

11. Grimes PE, Yamada N, Bhawan J (2005) Light microscopic, immunohistochemical, and ultrastructural alterations in patients with melasma Am J Dermatopathol 27: 96-101.

12. Kim JE, Chang SE, Yeo UC, Haw S, Kim H (2013) Histopathological study of the treatment of melasma lesions using a low-fluence Q-switched 1064-nm neodymium:yttrium-aluminium-garnet laser. Clin Exp Dermatol 38: 167-171.

13. Sanchez NP, Pathak MA, Sato S, Fitzpatrick TB, Sanchez JL, et al. (1981) Melasma: a clinical, light microscopic, ultrastructural, and immunofluorescence study. J Am Acad Dermatol 4: 698-710.

14. Lee DJ, Park KC, Ortonne JP, Kang HY (2012) Pendulous melanocytes: characteristic feature of melasma and how it may occur. $\mathrm{Br} \mathrm{J}$ Dermatol 166 : 684-686.

15. Inomata S, Matsunaga Y, Amano S, Takada K, Kobayashi K, et al. (2003) Possible involvement of gelatinases in basement membrane damage and wrinkle formation in chronically ultraviolet B-exposed hairless mouse. J Invest Dermatol 120: 128-134.

16. Luger TA, Schwarz T (1990) Evidence for an epidermal cytokine network. J Invest Dermatol 95: 100S-104S.

17. Imokawa G, Miyagishi M, Yada Y (1995) Endothelin-1 as a new melanogen: coordinated expression of its gene and the tyrosinase gene in UVB-exposed human epidermis. J Invest Dermatol 105: 32-37.

18. Schauer E, Trautinger F, Köck A, Schwarz A, Bhardwaj R, et al. (1994) Proopiomelanocortin-derived peptides are synthesized and released by human keratinocytes. J Clin Invest 93: 2258-2262.

19. Smit N, Le Poole I, van den Wijngaard R, Tigges A, Westerhof W, et al. (1993) Expression of different immunological markers by cultured human melanocytes. Arch Dermatol Res 285: 356-365.

20. Tomita $\mathrm{Y}$, Iwamoto M, Masuda T, Tagami H (1987) Stimulatory effect of prostaglandin E2 on the configuration of normal human melanocytes in vitro. $J$ Invest Dermatol 89: 299-301.

21. Imokawa G, Yada Y, Morisaki N, Kimura M (1998) Biological characterization of human fibroblast-derived mitogenic factors for human melanocytes. Biochem J $330: 1235-1239$

22. Grichnik JM, Burch JA, Burchette J, Shea CR (1998) The SCF/KIT pathway plays a critical role in the control of normal human melanocyte homeostasis. $J$ Invest Dermatol 111: 233-238.

23. Kim EH, Kim YC, Lee ES, Kang HY (2007) The vascular characteristics of melasma. J Dermatol Sci 46: 111-116.

24. Kang HY, Bahadoran P, Suzuki I, Zugaj D, Khemis A, et al. (2010) In vivo reflectance confocal microscopy detects pigmentary changes in melasma at a cellular level resolution. Exp Dermatol 19: e228-233.

25. Passeron T (2013) Long-lasting effect of vascular targeted therapy of melasma. J Am Acad Dermatol 69: e141-142.

26. Kim EJ, Park HY, Yaar M, Gilchrest BA (2005) Modulation of vascular endothelial growth factor receptors in melanocytes. Exp Dermatol 14: 625-633.

27. Jo HY, Kim CK, Suh IB, Ryu SW, Ha KS, et al. (2009) Co-localization of inducible nitric oxide synthase and phosphorylated Akt in the lesional skins of patients with melasma. J Dermatol 36: 10-16.

28. Kang HY, Hwang JS, Lee JY, Ahn JH, Kim JY, et al. (2006) The dermal stem cell factor and c-kit are overexpressed in melasma. Br J Dermatol 154: 1094-1099.

29. Ferrara N (2010) Binding to the extracellular matrix and proteolytic processing: two key mechanisms regulating vascular endothelial growth factor action. Mol Biol Cell 21: 687-690.

30. Bastaki M, Nelli EE, Dell'Era P, Rusnati M, Molinari-Tosatti MP, et al. (1997) Basic fibroblast growth factor-induced angiogenic phenotype in mouse endothelium. A study of aortic and microvascular endothelial cell lines. Arterioscler Thromb Vasc Biol 17: 454-464 
Citation: Kwon SH, Park KC (2014) Clues to the Pathogenesis of Melasma from its Histologic Findings. Pigmentary Disorders 1:141. doi: 10.4172/ JPD.1000141

31. Na Jl, Choi SY, Yang SH, Choi HR, Kang HY, et al. (2013) Effect of tranexamic acid on melasma: a clinical trial with histological evaluation. J Eur Acad Dermatol Venereol 27: 1035-1039.

32. Iddamalgoda A, Le QT, Ito K, Tanaka K, Kojima H, et al. (2008) Mast cell tryptase and photoaging: possible involvement in the degradation of extra cellular matrix and basement membrane proteins. Arch Dermatol Res 300 Suppl 1: S69-76.

33. Lavker RM, Kligman AM (1988) Chronic heliodermatitis: a morphologic evaluation of chronic actinic dermal damage with emphasis on the role of mast cells. J Invest Dermatol 90: 325-330.
34. Grimbaldeston MA, Simpson A, Finlay-Jones JJ, Hart PH (2003) The effect of ultraviolet radiation exposure on the prevalence of mast cells in human skin. $\mathrm{Br}$ J Dermatol 148: 300-306.

35. Gonzalez S, Moran M, Kochevar IE (1999) Chronic photodamage in skin of mast cell-deficient mice. Photochem Photobiol 70: 248-253.

36. Crivellato E, Nico B, Ribatti D (2008) Mast cells and tumour angiogenesis: new insight from experimental carcinogenesis. Cancer Lett 269: 1-6. 